\title{
Rossby numbers of fully convective and partially convective stars
}

\author{
Natália R. Landin ${ }^{1}$ and Luiz T. S. Mendes ${ }^{2}$ \\ ${ }^{1}$ Campus UFV Florestal - Universidade Federal de Viçosa, \\ CEP 35690-000, Florestal-M.G., Brazil \\ email: nlandin@ufv.br \\ ${ }^{2}$ Dept. de Engenharia Eletrônica, Universidade Federal de Minas Gerais, \\ CEP 31270-901, Belo Horizonte-M.G., Brazil \\ email: luiztm@cpdee.ufmg.br
}

\begin{abstract}
In this work, we investigate the stellar magnetic activity in the theoretical point of view, through the use of stellar structure and evolution models. We present theoretical values of convective turnover times and Rossby numbers for low-mass stars, calculated with the ATON stellar structure and evolution code. We concentrate our analysis on fully convective and partially convective stars motivated by recent observations of X-ray emission of slowly rotating fully convective stars, which suggest that the presence of a tachocline is not a central key for magnetic fields generation. We investigate the behavior of the convective turnover time evolution, as well as its radial profile inside the star. A discussion about the location where the convective turnover time is calculated in the stellar interior is also addressed. Our theoretical results are compared to observational data from low-mass stars.
\end{abstract}

Keywords. stars: rotation, convection, stars: activity, stars: magnetic fields.

\section{Introduction}

Stars of different spectral types and ages host large-scale magnetic fields, as evidenced by observable phenomena like star spots, flares, chromospheric and coronal emissions, all of which express in some way stellar magnetic activity. Activity in late-type stars is well correlated with rotation, but the activity-rotation correlation is usually better described in terms of the Rossby number $R_{o}$, defined as the ratio of the rotational period $P_{\text {rot }}$ to the convective turnover time $\tau_{c}\left(R_{o}=P_{\text {rot }} / \tau_{c}\right)$. Though $\tau_{c}$ can only be assessed theoretically, by using stellar models $\left(\tau_{c}=\alpha H_{p} / v_{c}\right.$, where $\alpha$ is the Mixing Length of Theory's paramenter, $H_{p}$ is the pressure scale height and $v_{c}$ is the convective velocity), it has been customary in the literature to use its empirical value, obtained as a function of the B- $V$ color index (Noyes et al. 1984). Recent observations by Wrigth \& Drake, 2016 of X-ray emission of slowly rotating fully convective stars indicate that partially and fully convective stars follow the same rotation-activity relationship and they operate very similar rotation-dependent dynamos. This implies that the presence of a tachoclina is not a central key for magnetic fields generation. In order to contribute to the understanding of the dynamo mechanism existing in fully and partially convective stars, we present theoretical values of $\tau_{c}$ and $R o$ for different stellar masses and ages calculated with the ATON stellar evolution code.

For a given stellar mass and age, the convective turnover time changes significantly depending on the location inside the star in which it is calculated. The location more commonly used in the literature is one half mixing length above the base of the convective zone, but this standard location seems to be not suitable for young very low mass stars. We, then, evaluate $\tau_{c}$ throughout the whole star at different evolutionary stages, in order 

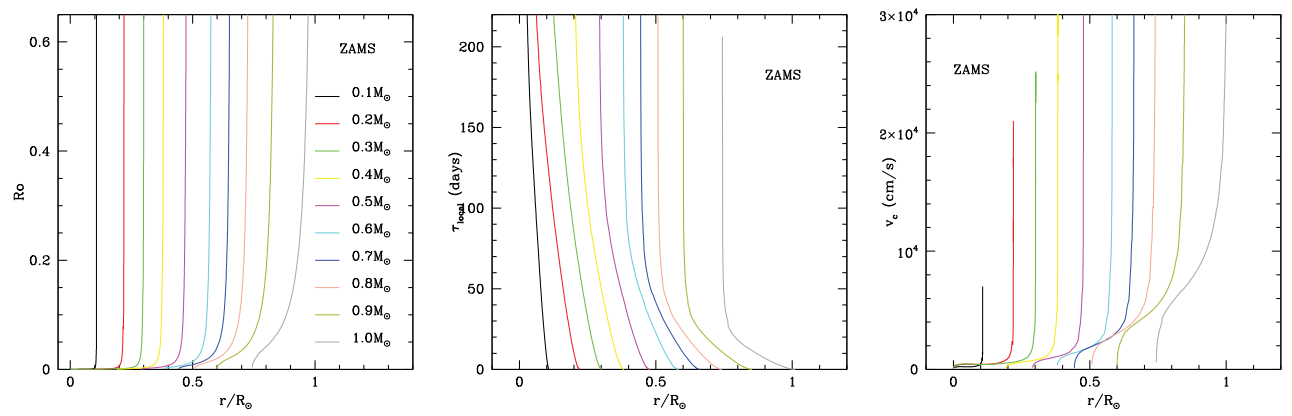

Figure 1. Profiles of $R o$ (left), $\tau_{c}$ (middle) and $v_{c}$ (right) for each stellar mass at the ZAMS.

to try to set this location in a alternative way. The convective turnover time profile is also investigated and our values are compared with those available in the literature. Finally, observational data from low-mass stars are used in order to test our theoretical results.

\section{Models}

In the version of ATON code we use in this work, convection is treated according to the Mixing Length of Theory (with $\boldsymbol{\alpha}=2$, the parameter that represents the convection efficiency) and non-grey surface boundary conditions is used. Rotating models were generated by assuming differential rotation (Mendes et al. 1999). The initial angular momentum of each model is obtained according to the Kawaler, 1987 relation

$$
J_{\text {kaw }}=1.566 \times 10^{50}\left(\frac{M}{M_{\odot}}\right)^{0.985} \quad \text { cgs. }
$$

The evolutionary tracks were computed in the mass range of 0.1-1.0 $M_{\odot}$. We adopted the solar chemical composition with $X=0.7155$ and $Z=0.0142$. More details about the physics of the models can be found in Landin et al. 2006.

\section{Results and Discussions}

First, we analyze how Rossby numbers vary inside the stars for all models at the ZAMS.

From the left panel of Fig. 1, we can see that the Rossby number profiles are very steep, mainly for lower mass stars. This behavior depends on the convective turnover time profiles in the stellar interiors at the ZAMS (middle panel of Fig. 1), which in their turn, depend on convective velocities shown in the right panel of Fig. 1. Masses increase from left $\left(0.1 M_{\odot}\right)$ to right $\left(1.0 M_{\odot}\right)$. In the left panel of Fig. 3, is the opposite.

Now, we analyze how $R o$ and $\tau_{c}$ vary as stars age. Plots of $R o, \tau_{c}$ and $v_{c}$ versus age, calculated at the standard location, are shown in Fig. $2 \dagger$. For main sequence and pre-main sequence models with $M<0.4 M_{\odot}$, the mixing length is so large that the place where $\tau_{c}$ should be calculated is larger than the stellar radius. In these cases, $\tau_{c}, v_{c}$ and $R o$ values are not calculated. Our results are in agreement with those by Kim \& Demarque, 1996.

Our calculations indicate that the usual location used to determine $R o$ is not suitable for young fully convective stars. We, then, have taken our $\tau_{c}$ values, computed at the standard location for ZAMS models with $M \geqslant 0.4 M_{\odot}$, and have evaluated them in terms of the stellar radius and the local $H_{p}$. After that, we have made a linear extrapolation

$\dagger$ Masses increase from bottom to top (left and right panel of Fig. 2 and middle and right panel of Fig. 3). In the middle panel of Fig. 2, is the opposite. Color figures in the online version. 

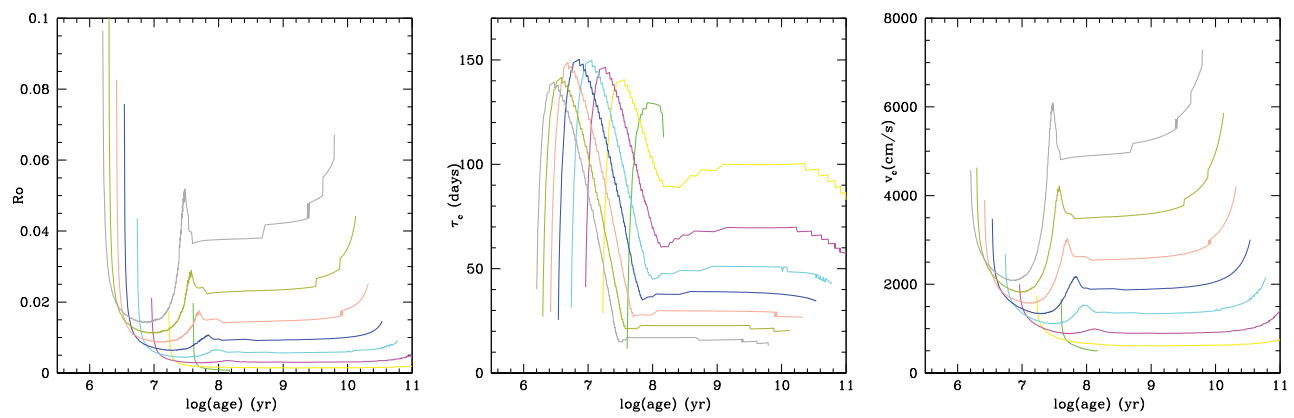

Figure 2. $R o, \tau_{c}$ and $v_{c}$ as a function of age and stellar mass.
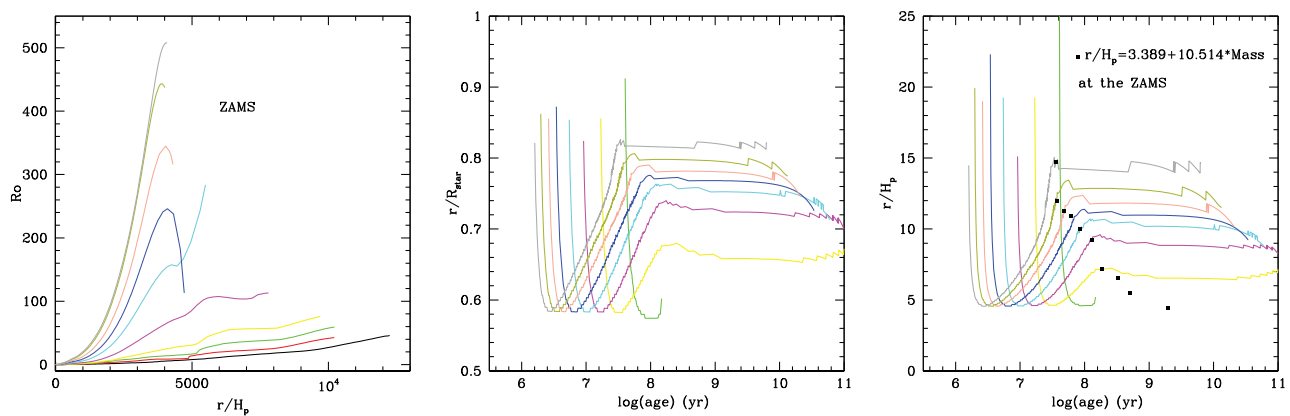

Figure 3. Ro profiles for all models at the ZAMS (left). Location, in terms of the stellar radius (middle) and $H_{p}$ (right), where the $R o$ is calculated as a function of mass and age.

of these $\tau_{c}$ values for lower mass stars and have found a suitable location to calculate $\tau_{c}$ as a funcion of mass (see the right panel of Fig. 3). In this way, we keep the equivalence between the standard location and the location derived in this work.

In the left panel of Fig. 3, we show Rossby number profiles at the ZAMS. According to Fig. 3 (middle), this derived location should be among 0.6 and $0.9 R_{\odot}$. Points in the right panel of Fig. 3 show where $R o$ is calculated in terms of $H_{p}$, at the ZAMS, for $M \geqslant 0.4 M_{\odot}$ (standard location) and for $M<0.4 M_{\odot}$ (extrapolation). As $\tau_{c}$ do not vary significantly during the main sequence (see middle panel of Fig. 2), we can use our new location to determine $\tau_{c}$ values for fully convective stars in this phase of evolution. By using $\tau_{c}$ values of stars with $M<0.4 M_{\odot}$ at these derived locations, we are able to reproduce the trend that fully convective stars follow in Wrigth \& Drake, 2016.

\section{Acknowledgments}

The authors thank D'Antona \& Mazzitelli for allowing them to use their ATON code. IAU 328 organizing committee is also acknowledged.

\section{References}

Kawaler, S. D. 1987, PASP, 99, 1322

Kim, Y. C. \& Demarque, P. S. 1996, ApJ, 457, 340

Landin N. R., Ventura, P. \& D'Antona, F., Mendes L. T. S., \& Vaz L. P. R.. 2006, A\&A, 456, 269

Mendes, L. T. S.., D’Antona, F., \& Mazzitelli, I. 1999, A\&A, 341, 174

Noyes, R. W., Hartmann, S.,W., Baliunas, S., Duncan, D. K., \& Vaughan, A. 1984, ApJ, 279, 763

Wright, N. J. \& Drake, J. J. 2016, Nature, 535, 526 